\title{
Multilevel effects of alcohol among early adolescents in an urban school district
}

\author{
Julie M. Croff ${ }^{1,2}$, Ronald B. Cox ${ }^{3}$, Isaac J. Washburn ${ }^{3}$, Chao Liu ${ }^{4}$, Clinton L. Broadbent ${ }^{5}$ \\ ${ }^{1}$ National Center for Wellness and Recovery, Oklahoma State University Center for Health Sciences, Tulsa, OK \\ ${ }^{2}$ Department of Rural Health, Oklahoma State University Center for Health Sciences. Tulsa, OK \\ ${ }^{3}$ Department of Human Development and Family Science, Oklahoma State University, Stillwater, OK \\ ${ }^{4}$ Department of Psychology, Cedarville University, Cedarville, $\mathrm{OH}$ \\ ${ }^{5}$ Department of Teacher Education \& Family Development, Southern Utah University, Cedar City, UT
}

\begin{abstract}
Aims: To examine how interpersonal interactions within and between the social networks formed by teachers, parents, students, and others shape the unique culture of the school, which in turn, reciprocally exerts a determining influence on each individual in the network.
\end{abstract}

Design: Cross-sectional study exploring whether factors associated with alcohol use at the individual level also exert influence on the culture of a school.

Setting: Twelve middle schools within an urban school district in the Midwestern United States.

Participants: Seventh grade students $(\mathrm{N}=1,620)$.

Measures: Lifetime alcohol use behaviors; mothers' and fathers' involvement in their child's education; and peer deviance.

Findings: The findings of this study suggest that as parents' involvement in education increases, and as peer deviance decreases, there are direct benefits to the child, and a protective effect for other children within the school when, in the aggregate, parental involvement increases and peer deviance decreases.

Conclusions: The effect of interventions to improve parent involvement and reduce peer deviance, with examinations at the individual-level and school-level, warrant future study.

\section{Introduction}

Alcohol use in adolescence remains a primary public health concern. By late adolescence, $15 \%$ of youth in the U.S. will meet criteria for dependence (Swendsen et al., 2012). Because alcohol use before age 15 is a significant predictor of dependence at some point in the lifetime (DeWit, Adlaf, Offord, \& Ogborne, 2000), continued development of alcohol-use prevention programs for adolescents is vital to the Institute of Medicine's 2030 Healthy People goal of reducing substance abuse in the population. This study examines whether factors known to influence adolescent alcohol use such as the interpersonal relationship with peers and parents will also influence the formation of a school culture that, in turn, can explain unique variance in individual alcohol use beyond the effect of the individuallevel factors.

Two ecological models suggest that student-level variables can interact to influence the cultural development of a school, Bronfenbrenner's ecodevelopmental model, and Waters' school ecological model. In the broader sense, Bronfenbrenner's model describes the interaction between ecological levels and the nesting of cultures and interpersonal relationships by community organization, including schools (Bronfenbrenner \& Ceci, 1994). Waters' model is an applied ecological model for schools that emphasizes schools as a physical structure or built environment in which student behaviors are affected by policies, and unique and intersecting cultural influences from interpersonal networks of peers, educators, and parents (Waters, Cross, \& Runions, 2009). Notably, Waters' school

Correspondence: Julie Croff, PhD, MPH, Oklahoma State University Center for Health Sciences, 1111 W 17th Street, Tulsa, OK 74107

Email: julie.croff@okstate.edu

Financial support: Dr Croff: Institutional Development Award (IDeA) from the National Institute of General Medical Sciences of the National Institutes of Health; R34 award from the National Institute on Drug Abuse [R34DA050237, R34DA05034-01S1 and R34DA05034-01S2] under grant number [P20GM109097]. Dr Cox: R15 award from the National Institute of Drug Abuse [R15DA049232].

Declaration of interest: None

Keywords: alcohol use, parent involvement, peer deviance, middle school, culture 
ecological model posits that the built school environment creates a community center wherein social networks act synergistically on students' abilities to connect to school, and that this connectedness directly influences student health and wellness (Waters et al., 2009). Unique school-level factors have been found to influence individual behavior, even when these same constructs at the individual-level do not influence individual behavior. For example, Cox and colleagues found that school-level SES influenced age of first use of alcohol but individual-level SES did not (2010).

Effects of peer interactions on adolescent substance use outcomes are well established in the literature, and supported as interpersonal relationships within ecological models. Very young adolescents (10 - 12 years old $)$ may be particularly susceptible to peer networks that use alcohol; knowing just one peer using alcohol places them at additional risk for use (Kelly et al., 2012). Moreover, popularity within peer networks is linked to increased alcohol use (Osgood et al., 2013). Peer influences may play a positive or negative role, with substance use among very young adolescents being considered a negative or deviant peer influence. Although suggested by Waters' ecological model, to date, studies have not examined an ecological or school-level effect of peer influence on individual-level alcohol use.

The influence of peers on alcohol use behavior may be due, at least in part, to peer norms. As has been identified in young adults, identification with one's peer group and perception of approval for drinking alcohol is crucial to the mechanism of action for norms on alcohol use (Reed, Lange, Ketchie, \& Clapp, 2007). Longitudinal analyses support that descriptive peer norms, or perceived normative behaviors among peers, mediate the relationship between alcohol use with peers (Brooks-Russell, Simons-Morton, Haynie, Farhat, \& Wang, 2014). School and community norms uniquely and cumulatively influence adolescent alcohol use (Roski et al., 1997). Notably, some have found that peer norms are more strongly correlated with adolescent alcohol use than parental involvement (Olds \& Thombs, 2001).

The influence of parenting behaviors on alcohol use is widely acknowledged (e.g., Spoth et al., 2009; Cox et al., 2013). One parenting intervention in secondary schools, a trial randomized at the school level, showed significant reductions in lifetime alcohol use at age 14.5 (Toumbourou, Gregg, Shortt, Hutchinson, \& Slaviero, 2013). National data indicate that a family's social capital protects adolescents from alcohol use (Dufur, Parcel, \& McKune, 2013). However, few studies have examined how parents may interact within ecological systems to create differential cultures that encourage or protect against alcohol use for all youth within the system. For instance, authoritative parenting has an effect at the neighborhood level such that a preponderance of parents practicing an authoritative style in a neighborhood influenced child outcomes over and above the influence of a child's particular parents (Fletcher, Darling, Steinberg, \& Dornbusch, 1995). The ecological effect of parenting has not yet been examined at the school level.

The interpersonal relationships with and between teachers and educational staff are responsible for key aspects of school culture that influence substance use. One factor associated with school culture is student perception of safety on campus; students' perception of safety is inversely associated with substance use within schools (Ennett, Flewelling, Lindrooth, \& Norton, 1997). Notably, education researchers share this conceptualization of safety as being a result of campus climate created by school personnel (Bradshaw et al., 2014).

\section{Objectives of the Current Study}

Together, these studies suggest that examination of secondlevel ecological influences on adolescent alcohol use are warranted. Parenting, negative peers influence, descriptive social norms, and school connectedness and safety may manifest at the school (level 2) and individual (level 1) level to protect against or encourage alcohol use. Factors that significantly influence alcohol use at the school level may be particularly important targets for future intervention.

\section{Methods}

\section{Sampling Procedures}

Seventh grade students from twelve public schools in an urban school district in the Midwestern United States were invited to participate in a study. A census of students was attempted and $98 \%$ participated in the study $(\mathrm{N}=1,736)$. Data was collected over a two-week period in May of 2009 using standardized self-report surveys in English or Spanish. Survey questions were read to students to avoid confounding due to literacy and to help maintain children on task. Students with learning disabilities severe enough to be exempt from annual end-of-instruction exams were excluded from the study. Missing values for the study variables were low ranging from $.1 \%$ to $4 \%$. Missing values were handled using Full Information Maximum Likelihood (FIML) estimation in Stata 14. This resulted in a final sample of 1,620 participants including $50.4 \%$ female $(n=817)$ with a mean age of 13.1. The sample was racially, or ethnically diverse (39\% Hispanic, 29\% Black, 20\% White, 12\% Other). The sample was primarily low-income with approximately $89 \%$ receiving free or reduced lunch (Schwerdtfeger Gallus, Shreffler, Merten, \& Cox Jr, 2015).

The Oklahoma State University's Office of Research Compliance, the school district's Planning, Research, and Evaluation Department, and the principal of each school sampled approved all study protocols, and granted permission to conduct the study. Parents were asked to opt children out as a form of consenting to participation in the study. Fewer than one percent of parents requested that their students not participate. Students each received a $\$ 5$ incentive for participation; and schools received \$200 for participation. Student participants gave assent to participate; they were informed of the goals of the study and nature of the questions. In ten of the schools, teachers administered the surveys during required classes. In two of the schools, surveys were administered to all students in the gymnasium or cafeteria by school administrators and teachers. Surveys for students who primarily spoke Spanish were delivered by one of the principal investigators (author RBC) and a Spanish-speaking graduate student. 
Lifetime Alcohol Use. Alcohol use was measured by a single item, "How old were you the first time you used alcohol?" The item was recoded into a dichotomous response: used or never used.

Perceived Mother and Father Educational Involvement. Mother and father involvement in school were each measured by four items that capture aspects of the student's perception of parental involvement in their schooling (e.g., My mother or mother figure: 1) makes sure I do my homework; 2) makes me feel good when I study or get good grades; 3) knows whether I go to school or not; 4) discusses report cards with me). Items were summed using a fourpoint scale ranging from strongly disagree to strongly agree so that higher scores indicate more involvement. In the current study, measures of internal consistency for mother and father involvement were strong $(\alpha=.82$, and .89 , respectively).

ATOD Descriptive Norms. Descriptive norms of Alcohol, Tobacco, and Other Drug use were measured with three items that capture perceptions of which substance use behaviors are typically performed at the school level (e.g., Do you agree that: 1) it is common for youth from your school to experiment with drugs?; 2) it is common for youth from your school to experiment with alcohol?; 3) it is common for youth from your school to smoke cigarettes?). Items were summed using a four-point scale ranging from strongly disagree to strongly agree so that higher scores indicate stronger norms. In the current study, measures of internal consistency for ATOD norms were strong $(\alpha=.90)$.

School Connectedness. School connectedness was measured with two items that capture aspects of interpersonal relationships with peers and school staff and a sense of belongingness at the school level (e.g., Regarding school, I feel: 1) close to people at this school; 2) like I am a part of this school). Items were summed using a four-point scale ranging from strongly disagree to strongly agree so that higher scores indicate a stronger sense of connectedness. In the current study, the measure of internal consistency for school connectedness was moderate $(\alpha=.68)$.

Peer Deviance. A negative construct of peer relationships, peer deviance was measured with six items that tapped into peers' deviant behaviors (e.g., Do you have close friends who: 1) have been in trouble with police?; 2) have stolen or damaged another person's belongings on purpose?; 3) belong to a gang?; 4) have skipped school?; 5) or plan to drop out of school?; 6) have become a teen parent?). Items had a binary yes/no response and were summed such that higher scores indicated greater deviance among close friends/peers. In the current study, the measure of internal consistency for peer deviance was strong $(\alpha=.80)$.

Safety. Students' perceptions of safety at school was measured by a single item, "Regarding school, I feel safe in my school." The item used a four-point scale ranging from strongly disagree to strongly agree, so that higher scores indicate higher feelings of safety. This item represents a unique overlap between school ecology and social support.

\section{Data Analytic Plan}

Because data are nested ( $7^{\text {th }}$ graders clustered in 12 schools), lifetime alcohol use was predicted using a multilevel logistic regression, specifically using the xtlogit command in Stata 16. We modeled a random intercept for alcohol use and tested for unique level 1 and level 2 effects controlling for gender, race, and perceived parental substance abuse. The initial model examined simultaneous effects of all variables to identify which variables were associated with lifetime alcohol use, and therefore belonged in the final model. Variables showing evidence of association with alcohol use were each centered on the school-mean for that variable. The school-mean was then subtracted from the original score for that variable to create two new variables, one representing the school level and one representing the student level. Created in this way these two variables decompose the variance in the association between our predictors and lifetime alcohol use into within and between schools (ICC = .02) (Enders \& Tofighi, 2007). These pairs of variables were then examined for their association with lifetime alcohol use in the same model with variance separated by level.

\section{Results}

The results of the simultaneous logistic regression predicting lifetime alcohol use are presented in Table 1. Both mother's and father's educational involvement at the individual and school level were negatively associated with having used alcohol. Perceived safety within schools and school connectedness were not significantly related to adolescent lifetime alcohol use. Increases in seeing ATOD use as normative and having more deviant peer associations were both significantly associated with an increased probability of having used alcohol. However, school connectedness did not explain any variance in lifetime adolescent alcohol use.

After dropping school connectedness from the model, a multilevel model was run to identify the influence of each set of variables at each level: school- and individual-level. Participant reports of mother's and father's interest in their education was a significant predictor at the school- and student-level. As the average level of mother's and father's interest in school increases at the school-level, the probability of having used alcohol for all students in that school decreases. ATOD norms did not show a significant effect at the school level, but did so at the individual level. Reporting that a close friend exhibited deviant behaviors was associated with lifetime alcohol use at both the school- and student-level. Feeling safe at school was only significantly associated with having used alcohol at the student-level. 
Table 1

Lifetime Alcohol Use at the Individual Level and as a Multilevel Model

1a. Simultaneous effects: logistic regression on lifetime alcohol use without controls

\begin{tabular}{lcccc}
\hline & $B$ & $S E$ & $Z$ & $p$ \\
\hline Dad educational involvement & -0.32 & 0.07 & -4.75 & 0.000 \\
Mom educational involvement & -0.41 & 0.10 & -4.30 & 0.000 \\
ATOD norms & 0.31 & 0.07 & 4.53 & 0.000 \\
School connectedness & 0.13 & 0.10 & 1.34 & 0.180 \\
Peer deviance & 2.04 & 0.19 & 10.78 & 0.000 \\
Safety & -0.14 & 0.07 & -1.91 & 0.060 \\
Intercept & 0.43 & 0.44 & 0.99 & 0.320 \\
\hline
\end{tabular}

1b. Individual effects of lifetime alcohol use by level with controls

\begin{tabular}{|c|c|c|c|c|}
\hline & $B$ & $S E$ & $Z$ & $p$ \\
\hline School level - dad educational involvement & -1.34 & 0.50 & -2.70 & 0.007 \\
\hline Student level - dad educational involvement & -0.55 & 0.06 & -9.20 & 0.000 \\
\hline Male gender & 0.17 & 0.11 & 1.58 & 0.114 \\
\hline African American/black & 0.32 & 0.17 & 1.90 & 0.057 \\
\hline Hispanic/Latino & 0.58 & 0.17 & 3.36 & 0.001 \\
\hline Asian/pacific islander & 0.13 & 0.33 & 0.39 & 0.699 \\
\hline Native American/Alaska native & 0.60 & 0.29 & 2.08 & 0.037 \\
\hline Other racial/ethnic group & 0.49 & 0.30 & 1.63 & 0.103 \\
\hline Mom alcohol problems & 0.16 & 0.09 & 1.87 & 0.061 \\
\hline Dad alcohol problems & 0.23 & 0.07 & 3.58 & 0.000 \\
\hline Intercept & 2.44 & 1.50 & 1.62 & 0.104 \\
\hline School level - mom educational involvement & -1.85 & 0.52 & -3.56 & 0.000 \\
\hline Student level - mom educational involvement & -0.85 & 0.09 & -9.73 & 0.000 \\
\hline Male gender & 0.17 & 0.11 & 1.58 & 0.113 \\
\hline African American/black & 0.53 & 0.17 & 3.19 & 0.001 \\
\hline Hispanic/Latino & 0.50 & 0.16 & 3.11 & 0.002 \\
\hline Asian/pacific islander & 0.06 & 0.32 & 0.19 & 0.851 \\
\hline Native American/Alaska native & 0.63 & 0.29 & 2.18 & 0.029 \\
\hline Other racial/ethnic group & 0.66 & 0.30 & 2.20 & 0.028 \\
\hline Mom alcohol problems & 0.03 & 0.09 & 0.36 & 0.720 \\
\hline Dad alcohol problems & 0.34 & 0.06 & 5.30 & 0.000 \\
\hline Intercept & 4.63 & 1.76 & 2.64 & 0.008 \\
\hline School level - ATOD norms & 0.26 & 0.41 & 0.64 & 0.522 \\
\hline Student level - ATOD norms & 0.64 & 0.06 & 9.88 & 0.000 \\
\hline Male gender & 0.16 & 0.11 & 1.45 & 0.146 \\
\hline African American/Black & 0.39 & 0.17 & 2.27 & 0.023 \\
\hline Hispanic/Latino & 0.71 & 0.17 & 4.10 & 0.000 \\
\hline Asian/Pacific Islander & 0.28 & 0.33 & 0.84 & 0.399 \\
\hline Native American/Alaska Native & 0.52 & 0.29 & 1.80 & 0.072 \\
\hline Other racial/ethnic group & 0.51 & 0.30 & 1.72 & 0.086 \\
\hline Mom alcohol problems & 0.08 & 0.09 & 0.93 & 0.355 \\
\hline Dad alcohol problems & 0.31 & 0.06 & 4.84 & 0.000 \\
\hline Intercept & -2.10 & 0.79 & -2.66 & 0.008 \\
\hline
\end{tabular}


1b. Individual effects of lifetime alcohol use by level with controls (continued)

\begin{tabular}{lcccc}
\hline & $B$ & $S E$ & $Z$ & $p$ \\
\hline School level - peer deviance & 3.30 & 0.77 & 4.29 & 0.000 \\
Student level - peer deviance & 2.54 & 0.18 & 13.73 & 0.000 \\
Male gender & 0.32 & 0.11 & 2.86 & 0.004 \\
African American/Black & 0.19 & 0.17 & 1.10 & 0.272 \\
Hispanic/Latino & 0.31 & 0.17 & 1.81 & 0.070 \\
Asian/Pacific Islander & 0.24 & 0.33 & 0.73 & 0.464 \\
Native American/Alaska Native & 0.20 & 0.30 & 0.67 & 0.502 \\
Other racial/ethnic group & 0.31 & 0.31 & 1.01 & 0.313 \\
Mom alcohol problems & 0.09 & 0.09 & 1.04 & 0.297 \\
Dad alcohol problems & 0.28 & 0.07 & 4.27 & 0.000 \\
Intercept & -2.60 & 0.31 & -8.35 & 0.000 \\
\hline
\end{tabular}

\section{Discussion}

This study explores how factors associated with alcohol use at the individual level may aggregate at the school level to create a protective benefit for individuals who do not have individually protective environments, therefore, with a sufficient and robust group of protective individual-level parenting influences, the culture of a school becomes protective against alcohol use. This follows Bronfenbrenner's and Waters' ecological models wherein two microsystem effects (e.g., parents and schools) interact to influence an outcome (Bronfenbrenner \& Ceci, 1994; Waters et al., 2009), in this case, lifetime alcohol use. Taken together, adolescent interpersonal relationships and descriptive norms impact the school's cultural environment, which in turn, influences individual use. Direct links to the school environment may operate through parent, and peer relationships (Leventhal and Brooks-Gunn, 2000). This study is among the first to demonstrate unique effects at the school-level of parental involvement in education, and ATOD norms within the school which will be explored in detail in the following paragraphs.

Mothers' and fathers' involvement in adolescent educational attainment was protective of lifetime alcohol use in this sample of $7^{\text {th }}$ grade students. Most notably, these interests had effects at the school-level, beyond the student most directly affected by parenting. The mechanism for this action is not well understood and warrants future examination. One might hypothesize that parents may influence other youth within the school through peer networks through heightened parental educational expectations and heightened parental monitoring of behaviors. Because alcohol use occurs in peer groups, a parent can have a positive influence on a group of students within a school system.

Adolescent perceptions of peer ATOD norms was significantly related to alcohol use at the individual-level, even when controlling for parental educational involvement and perceived school safety. However, these ATOD norms acted only at the individual level. Previous work in this area suggests that descriptive norms may have power only when an individual associates with the group of interest (Reed et al., 2007). This result suggests that social norms marketing approaches may not work for this social group. Other research supports the role of ATOD norms for influencing substance use behaviors in adolescents. Given that adolescents are more likely to respond to pro-substance use norms of high-status peers (Teunissen et al., 2012), interventions developed to address this may have some effect at the individual-level.

Perceptions of peer deviance were significantly related to alcohol use at the individual-level and at the school-level even after controlling for mother's and father's educational involvement. Peer networks, including deviant peers, have a strong influence in very early adolescence. Deviant peer behaviors impacting the substance use culture of a school should be the topic of future research studies. Interventions and programs at the peer-network level show promise for reducing alcohol use (Mason et al., 2015), and may be beneficial for changing heavy using school environments.

The results of this study should foster additional research. The cross-sectional nature, and focus on lifetime use rather than temporally proximal alcohol use, may limit generalizability of the results. Future studies should work to replicate the findings with additional schools and longitudinal data. More schools will provide more power to find an effect and longitudinal data will help with time sequencing problems. Future studies should examine parents' behaviors, rather than adolescent perceptions of parenting. Moreover, factors that influence parental interest in their children's educational attainment warrant additional examination. Over several waves of data collection in one longitudinal study, neighborhood quality and familial poverty in elementary school predicted lower levels of parental involvement during adolescence (Hampson et al., 2016). There may be sufficient evidence to suggest that parenting programs that encourage educational attainment of adolescents have widespread impact on health and substance use behaviors, particularly because second level effects are difficult to find with as few as twelve schools. These results suggest that variables aggregated at the school level may be exerting a much stronger effect than previously thought. This finding is heartening for prevention programming because 
fewer than $100 \%$ of parents must change their behavior in order to create a second-level effect protective of all children. Parent educational engagement programs need not target all parents, as strong results at the school-level may come from a core team of key parent leaders.

\section{References}

Bradshaw, C. P., Waasdorp, T. E., Debnam, K. J., \& Johnson, S. L. (2014). Measuring school climate in high schools: A focus on safety, engagement, and the environment. Journal of School Health, 84, 593604. https://doi.org/10.1111/josh.12186

Bronfenbrenner, U., \& Ceci, S. J. (1994). Nature-nuture reconceptualized in developmental perspective: A bioecological model. Psychological Review, 101(4), 568-586. https://doi.org/10.1037/0033-295x.101.4.568

Brooks-Russell, A., Simons-Morton, B., Haynie, D., Farhat, T., \& Wang, J. (2013). Longitudinal relationship between drinking with peers, descriptive norms, and adolescent alcohol use. Prevention Science: The Official Journal of the Society for Prevention Research, 15. https://doi.org/10.1007/s11121-013-0391-9

Cox, R. B., Blow, A. J., Maier, K. S., \& Cardona, J. R. P. (2010). Covariates of substance-use initiation for Venezuelan youth: Using a multilevel approach to guide prevention programs. Journal of Studies on Alcohol and Drugs, 71(3), 424-433. https://doi.org/10.15288/jsad.2010.71.424

DeWit, D. J., Adlaf, E. M., Offord, D. R., \& Ogborne, A. C. (2000). Age at first alcohol use: A risk factor for the development of alcohol disorders. The American Journal of Psychiatry, 157(5), 745-750. https://doi.org/10.1176/appi.ajp.157.5.745

Dufur, M. J., Parcel, T. L., \& McKune, B. A. (2013). Does capital at home matter more than capital at school? The case of adolescent alcohol and marijuana use. Journal of Drug Issues, 43(1), 85-102. https://doi.org/10.1177/0022042612462220

Enders, C. K., \& Tofighi, D. (2007). Centering predictor variables in cross-sectional multilevel models: A new look at an old issue. Psychological Methods, 12(2), 121138. https://doi.org/10.1037/1082-989x.12.2.121

Ennett, S. T., Flewelling, R. L., Lindrooth, R. C., \& Norton, E. C. (1997). School and neighborhood characteristics associated with school rates of alcohol, cigarette, and marijuana use. Journal of Health and Social Behavior, 38(1), 55-71. https://doi.org/10.2307/2955361

Fletcher, A. C., Darling, N. E., Steinberg, L., \& Dornbusch, S. (1995). The company they keep: Relation of adolescents' adjustment and behavior to their friends' perceptions of authoritative parenting in the social network. Developmental Psychology, 31(2), 300-310. https://doi.org/10.1037/0012-1649.31.2.300

Hampson, S. E., Andrews, J. A., Barckley, M., Gerrard, M., \& Gibbons, F. X. (2016). Harsh environments, life history strategies, and adjustment: A longitudinal study of Oregon youth. Personality and Individual Differences, $\quad 88, \quad 120-124$. https://doi.org/10.1016/j.paid.2015.08.052

Kelly, A. B., Chan, G. C. K., Toumbourou, J. W., O'Flaherty, M., Homel, R., Patton, G. C., \& Williams, J. (2012). Very young adolescents and alcohol: Evidence of a unique susceptibility to peer alcohol use. Addictive Behaviors, $\quad 37(4), \quad 414-419$. https://doi.org/10.1016/j.addbeh.2011.11.038

Leventhal, T., \& Brooks-Gunn, J. (2000). The neighborhoods they live in: The effects of neighborhood residence on child and adolescent outcomes. Psychological Bulletin, 126(2), 309337. https://doi.org/10.1037/0033-2909.126.2.309

Mason, M., Light, J., Campbell, L., Keyser-Marcus, L., Crewe, S., Way, T., . . . McHenry, C. (2015). Peer network counseling with urban adolescents: A randomized controlled trial with moderate substance users. Journal of Substance Abuse Treatment, 58, 16-24. https://doi.org/10.1016/j.jsat.2015.06.013

Olds, R. S., \& Thombs, D. L. (2001). The relationship of adolescent perceptions of peer norms and parent involvement to cigarette and alcohol use. Journal of School Health, 71(6), 223$228 . \quad$ https://doi.org/10.1111/j.17461561.2001.tb01322.x

Osgood, D. W., Ragan, D. T., Wallace, L., Gest, S. D., Feinberg, M. E., \& Moody, J. (2013). Peers and the emergence of alcohol use: Influence and selection processes in adolescent friendship networks. Journal of Research on Adolescence, 23(3), 500-512. https://doi.org/10.1111/jora.12059

Reed, M. B., Lange, J. E., Ketchie, J. M., \& Clapp, J. D. (2007). The relationship between social identity, normative information, and college student drinking. Social Influence, 2(4), 269-294. https://doi.org/10.1080/15534510701476617

Roski, J., Perry, C. L., McGovern, P. G., Williams, C. L., Farbakhsh, K., \& Veblen-Mortenson, S. (1997). School and community influences on adolescent alcohol and drug use. Health Education Research, 12(2), 255-266. https://doi.org/10.1093/her/12.2.255

Schwerdtfeger Gallus, K. L., Shreffler, K. M., Merten, M. J., \& Cox, R. B. (2015). Interpersonal trauma and depressive symptoms in early adolescents: Exploring the moderating roles of parent and school connectedness. The Journal of Early Adolescence, 35(7), 990-1013. https://doi.org/10.1177/0272431614548067

Spoth, R., Trudeau, L., Guyll, M., Shin, C., \& Redmond, C. (2009). Universal intervention effects on substance use among young adults mediated by delayed adolescent substance initiation. Journal of Consulting and Clinical Psychology, 77(4), 620-632. https://doi.org/10.1037/a0016029

Swendsen, J., Burstein, M., Case, B., Conway, K., Dierker, L., He, J., \& Merikangas, K. (2012). Use and Abuse Of Alcohol And Illicit Drugs In US Adolescents: Results Of The National Comorbidity Survey - Adolescent Supplement. Archives of General Psychiatry, 69, 390398.

https://doi.org/10.1001/archgenpsychiatry.2011.1503

Teunissen, H. A., Spijkerman, R., Prinstein, M. J., Cohen, G. L., Engels, R. C., \& Scholte, R. H. (2012). Adolescents' conformity to their peers' pro-alcohol and anti-alcohol norms: the power of popularity. Alcoholism, Clinical and Experimental Research,36(7), 1257-1267. https://doi.org/10.1111/j.1530-0277.2011.01728.x 
Toumbourou, J. W., Gregg, M. E. D., Shortt, A. L., Hutchinson, D. M., \& Slaviero, T. M. (2013). Reduction of adolescent alcohol use through family-school intervention: A randomized trial. Journal of Adolescent

Health, 53(6),

778-784.

https://doi.org/10.1016/j.jadohealth.2013.07.005

Waters, S., Cross, D., \& Runions, K. (2009). Social and ecological structures supporting adolescent connectedness to school: A theoretical model. The Journal of School Health, 79, 516-524. https://doi.org/10.1111/j.1746-1561.2009.00443.x 